\title{
1 Crops and the seed mass-seed output trade-off in plants
}

3 Adam R. Martin ${ }^{1, *}$

$4 \quad{ }^{1}$ Department of Physical and Environmental Sciences, University of Toronto

5 Scarborough, Canada.

$6 *$ Author correspondence: adam.martin@utoronto.ca

\section{Abstract}

9 A trade-off between seed mass (SM) and seed output (SO) defines a central axis

10 of ecological variation among plants, with implications for understanding both plant trait

11 evolution and plant responses to environmental change. While an observed negative SM-

12 SO relationship is hypothesized to reflect universal constraints on resource allocation in

13 all plants, domestication has likely fundamentally altered this relationship. Using a

14 dataset of SM and SO for 41 of the world most widespread crops and 1,190 wild plant

15 species, coupled with observational data on these traits in soy (Glycine max) and maize

16 (Zea mays), I show that domestication has systematically rewired SM-SO relationships in

17 crops. Compared to wild plants, virtually all crops express a higher SM for a given SO;

18 this domestication signature is especially prominent in seed crops, and also influences the

19 phylogenetic signal in SM and SO. In maize these traits have become positively related

20 likely due to simultaneous selection for greater SM and SO, while in soy these traits have

21 become decoupled likely due to primary selection for SM only. Evolved relationships

22 between SM and SO in plants have been disrupted by both conscious and unconscious

23 artificial selection, which represents a key aspect of how the functional biology of crops

24 differ fundamentally from wild plants along "universal" plant trait spectra. 


\section{Introduction}

28 Seed mass and seed output as a critical dimension of plant functional ecology

29 Differences in seed traits including mass, output, shape, dispersal, biochemical

30 constitution, and dormancy have come to represent critical axes of life-history variation

31 among plant species worldwide (Westoby et al. 1996, Moles et al. 2005, Diaz et al.

32 2016). Understanding and quantifying variation in these seed traits among terrestrial plant

33 species has been central in better understanding a range of large-scale processes including

34 the evolution of angiosperms and plant biogeography (e.g. Westoby et al. 1992,

35 Thompson et al. 1993, Westoby et al. 1996, Moles and Westoby 2004, Moles et al. 2005),

36 as well as multiple aspects of plant life history strategy including dispersal ability, stature,

37 seedling competitiveness and survivorship, plant- and species responses to disturbance,

38 colonization ability, and persistence of seeds in soils (Thompson et al. 1993, Westoby et

39 al. 1996, Leishman et al. 2000, Moles and Westoby 2003, 2004). Of the seed traits

40 explored in the comparative plant sciences, seed mass (SM) has received the most

41 research attention (cf. Kattge et al. 2011), with comparative analyses including thousands

42 of plant species indicating that SM varies by up to 6 orders of magnitude across species

43 (Westoby et al. 2002, Moles and Westoby 2003). When considered independently or

44 alongside other leaf-, stem- and whole-plant traits, variation in SM across species

45 represents a key trait defining the functional ecology of plants worldwide (e.g. Westoby

46 1998, Cornelissen 1999, Westoby et al. 2002, Diaz et al. 2016).

In plant ecology, a long-recognized trade-off between SM and seed output (SO)

49 pertains to plant reproduction strategies (Harper et al. 1970, Smith and Fretwell 1974,

50 Lloyd 1987, Westoby et al. 2002, Moles et al. 2004). Under conditions of finite

51 resources, theory and observation suggest plant species differentiate from one another

52 along a SM-SO axis that is hypothesized to optimize reproductive fitness and success,

53 given a certain set of environmental conditions (Sadras 2007). In the simplest conceptual

54 terms, the endpoints of the SM-SO trade-off are defined by plants allocating resources to

55 a small number of large seeds, vs. plants that allocate resources to a large number of very

56 small seeds; the wide variation that exists in between these conceptual points reflects

57 "optimized" solutions to resource allocation (Sadras 2007). Similar to the evidence that 
58 supports the existence of universal trade-offs (or correlations) among other plant

59 functional traits (e.g. Wright et al. 2004), global databases including trait values from

60 thousands of species can be used to define a "universal SM-SO" trade-off that exists

61 among plants globally. The shape of this trade-off reflects evolutionarily defined

62 constraints on possible combinations of SM and SO that can (or are most likely to) occur

63 across non-domesticated plant species (hereafter referred to as "wild plants").

Seed mass and seed output trade-offs and crops

Historical analyses(Meyer et al. 2012) coupled with recent observational and experimental studies, have refined our understanding of how plant trait variation and correlations have been fundamentally altered by crop domestication (Meyer et al. 2012, Milla et al. 2014, Martin et al. 2017). While the suites of traits that are under intentional and unintentional artificial selection is wide - including whole-plant, leaf-, and root traits (e.g. Milla et al. 2014) - plant yield components including SM and/ or SO have been under the most intensive selection (Sadras 2007).

Assuming that crops have been selected for increased SM and SO leads to the

74 hypothesis ("Hypothesis 1) that, as compared to a "global SM-SO trade-off", artificial 75 selection results in all crops expressing a higher SM for a given SO (or vice versa) as 76 compared to wild plants. Additionally though, artificial selection directly targets SM and SO only for a certain group of crops such as cereals (e.g. wheat, rice, and maize), oil seed crops, or legumes including soy and other pulses. Therefore, one may also hypothesize ("Hypothesis 2") that for a given crop species, the degree of divergence away from a global SM-SO trade-off differs according to the plant organ under selection, with seed

81 crops showing the strongest divergences on average.

Yet even for crops under selection for seeds, within-species SM-SO trade-offs

83 may differ considerably according to plant growth form and reproductive strategy. In

84 certain crops including maize - one of the crops employed in my analysis here - both

85 increased SM (i.e. mean kernel mass) (Hufford et al. 2012) and SO (i.e. mean kernels per

86 plant)(Brown et al. 2011) have been simultaneously targeted by artificial selection. This

87 leads to the hypothesis (Hypothesis 3) that, counter to a global SM-SO trade-off, crops

88 such as maize express a positive SM-SO correlation. Alternatively, genome sequencing 
89 indicates that increased SM in soybean - also employed in my analysis here - has been

90 targeted during artificial selection (Liu et al. 2007, Zhou et al. 2015), while the number of

91 inflorescences and ultimately SO remains plastic, and largely determined by local

92 resource availability and concomitant plant growth rates (Andrade et al. 2005). This leads

93 to the hypothesis (Hypothesis 4) that in soy, covariation along an intraspecific SM-SO

94 trade-off would be weak or potentially non-existent. Here, I used a large dataset of SM

95 and SO from 1,190 wild plant species and 41 of the world's most widespread crops,

96 derived from both functional trait databases and field studies on maize and soy, in order

97 to test these four complementary hypotheses.

\section{Methods}

Generating a global SM-SO trade-off with functional trait data

Data for both SM and SO were acquired from a structured enquiry submitted to

102 the TRY Functional Trait Database (Kattge et al. 2011). We specifically requested

103 information on trait ID 26 ("seed dry mass", corresponding to SM) and trait ID 131

104 ("seed number per plant", corresponding to SO). This request returned $n=117,882$ and

$105 n=9,292$ observations for SM and SO respectively. All statistical analyses were then

106 performed using R version 3.4.0 (R Foundation for Statistical Computing, Vienna,

107 Austria). First, for each observation I used the 'TPL' function in the "'Taxonstand' R

108 package (Cayuela et al. 2017) in order to cross-reference all species, genus, and family

109 identities with The Plant List and resolve all synonyms or errors. Once taxonomy checks

110 were complete, species-level mean values for both SM and SO were then calculated.

111 This list of species was then cross-referenced with a list of the world's crop

112 species reported by the Food and Agricultural Organization of the United Nations (FAO)

113 (FAO 2018), which was refined to species-level taxonomy by Martin and Isaac (2015).

114 This procedure led to identification of $n=38$ crop species which had both paired SM-SO

115 data in TRY, and have been identified by the FAO as a commodity species. I therefore

116 supplemented the TRY dataset with species mean SM and SO values extracted from the

117 literature for a number of additional common crops including soy (Glycine max(Hayes et

118 al. 2018)), sunflower (Helianthus annuus(Libenson et al. 2002), rice (Oryza sativa(Wang

119 et al. 2008)), and maize (Zea mays(Maddonni and Otegui 2006)); data for SM and SO 
120 was also sought for all additional crops listed by Martin and Isaac (2015) which were not

121 in TRY, but this data was not available in peer-reviewed literature. Therefore in sum, this

122 data consolidation process resulted in paired SM-SO data for $n=1231$ species in total.

123 Each of the 41 crop species were then classified broadly according to the main

124 commercial portion of the plant as one of: i) "seed plants" which are harvested for seeds

125 ( $n=17$ crop species); ii) "tree crops" which are harvested for timber or as ornamental

126 species ( $n=3$ crop species); iii) "other crops" which are harvested for other plant parts

127 including leaves, roots, or large inflorescences ( $n=21$ crop species); or iv) "wild species"

128 which are non-domesticated plants ( $n=1190$ species).

\section{Crops along an SM and SO trade-off}

131 Analyses using the 'fitdist' function in the 'fitdistrplus' R package (Delignette-

132 Muller and Dutang 2015) indicating that both the SM and SO datasets were better

133 described by normal or log-normal distributions (as per lower log-likelihood scores;

134 Table 1), so log-transformed data was used in all subsequent analyses. I first fit a

135 standardized major axis regression (SMA) model to the entire log-SM and log-SO, in

136 order to test for the presence of a "global SM-SO trade-off". This SMA was implemented

137 in using the 'sma' function in the 'smatr' R package(Warton et al. 2012), with 95\%

138 confidence limits surrounding the overall model generated through bootstrapping.

139 All SMA model residuals associated with each species were then extracted, and

140 significant differences in residuals among the four different plant types were evaluated.

141 Due to unequal sample sizes and non-independence of data points owing to phylogenetic

142 structure in the data (see below), this test was performed as a linear mixed effects model

143 using the 'Ime' function in the 'nlme' R package(Pinheiro et al. 2016). Specifically, in

144 this model SMA residuals were predicted as a function of plant type (as a fixed effect),

145 while accounting for genus identity nested within family identity (as random effects). In

146 addition to assessing overall significance of the plant type term (i.e. the fixed effect), I

147 calculated mean ( \pm S.E.) SMA residuals for each plant type using the 'lsmeans' function

148 in the 'lsmeans' R package (Lenth 2016), and assessed the pairwise differences in mean

149 SMA model residuals among all four plant types using a Tukey post-hoc tests (also

150 implemented with the 'Ismeans' function). Post-hoc tests based on this linear mixed- 
151 effect model were considered significant when assessed against a Bonferonni-corrected $152 p$-value of 0.008 .

153

Phylogenetic signal in SM and SO

155 A phylogenetic tree was constructed for the entire $n=1,231$ species using

156 Phylomatic (Webb and Donoghue 2005) to generate a phylogenetic tree, based on the 157 Angiosperm Phylogeny Group megatree ("R20120829.new”). The BLADJ algorithm in

158 Phylocom(Webb et al. 2008) was used to estimate phylogenetic branch lengths according

159 to clade ages based on fossil records (Wikstrom et al. 2001) which were updated by

160 Gastauer and Meira-Neto (2016). Unresolved evolutionary relationships were treated as 161 polytomies.

162 Phylogenetic signal in log-SM and log-SO across the entire phylogeny was then 163 quantified as Pagel's $\lambda$ (Pagel 1999). For this analysis, a Pagel's $\lambda$ value equal to 0

164 represents instances of no phylogenetic signal (i.e. where evolution of SM and/or SO is 165 entirely independent of phylogeny), and Pagel's $\lambda$ values of 1 represent instances where a 166 phylogeny perfectly predicts trait data (i.e. where evolution of SM and/or SO perfectly 167 matches a Brownian model of trait evolution) (Pagel 1999). Values of Pagel's $\lambda$ were 168 calculated using the 'phylosig' function in the 'phytools' R package (Revell 2012).

169 Significance tests for were performed as randomization tests (with $n=1000$

170 randomizations used), where SM and SO data were randomly shuffled across the 171 phylogeny, and Pagel's $\lambda$ was recalculated on each randomized dataset; phylogenetic 172 signal was considered statistically significant if the observed Pagel's $\lambda$ fell within the 173 upper $95 \%$ of this randomized distribution.

174 In order to assess if the presence of crops influenced phylogenetic signal in SM

175 and SO, I then recalculated Pagel's $\lambda$ with crop species removed from the dataset (where

$176 n=1,190$ in this reduced phylogeny and trait dataset). Specifically, if Pagel's $\lambda$ was to

177 increase when crops were removed from the phylogeny, this could be interpreted as crops

178 reducing the strength of the phylogeny signal in SM or SO. All trait-phylogeny

179 relationships were also graphed visually using the 'plotTree.wBars' function in the

180 'phytools' R package (Revell 2012). 
183 I employed two crop species-specific datasets, both derived from field studies, to

184 evaluate if soy and maize expressed an SM-SO trade-off that was consistent with a global

185 pattern (i.e. based on the $n=1,231$ species dataset). Soy data was taken from a published

186 field study focused on soy leaf economics traits that was conducted on a 30-year old

187 experimental farm in Guelph, Canada $\left(43^{\circ} 32^{\prime} \mathrm{N}, 80^{\circ} 12^{\prime} \mathrm{W}\right)$ (Hayes et al. 2018). This

188 study provided paired plant-level SM and SO data for $n=45$ soy plants (detailed in (Hayes

189 et al. 2018)). Maize data was taken from two different field studies conducted at the same

190 site, where paired SM and SO data was directly available (Maddonni and Otegui 2006,

191 Mayer et al. 2012). Specifically, $n=26$ paired maize SM-SO observations were available

192 in Table 1 of (Maddonni and Otegui 2006), while $n=8$ paired maize SM-SO data were

193 taken from Table 1 of (Mayer et al. 2012). Data from both maize studies were derived

194 from field experiments at the same site (the Experimental Station of the National Institute

195 of Agricultural Technology) in Pergamino, Argentina (33 56' S, 60 $34^{\prime} \mathrm{W}$ ). I then used

196 fit a SMA model (as described above) to both soy and maize datasets separately to

197 describe intraspecific SM and SO patterns, and where SMA models were significant,

198 95\% confidence limits were generated through bootstrapping with replacement (with

1991000 replicates used) implemented with the 'bootstrap' function in the 'modelr' R

200 package (Wickham 2017).

201

202 Data availability

203 The compiled database of $n=1,231$ plant species used in my analyses here

204 (presented in Figs. 1 and 4) are available as individual datasets in the TRY Functional

205 Trait Database. Compiled data on soy and maize (presented in Fig. 3) are available upon

206 request from the author, or in the original publications as cited in the methods.

208 Results

209 Crops along a global seed mass seed output trade-off

210 Data from $n=1231$ species across 517 genera and 92 families demonstrate the

211 presence of a SM-SO trade-off in plants globally, with a standardized major axis (SMA)

212 regression slope of $-0.86\left(95 \%\right.$ C.I. $=-0.91,-0.82$, SMA $r^{2}=0.06, p<0.0001$; Fig. 1$)$. Of 
213 the 41 crop species represented in the SM-SO dataset, 90.2\% (37 crop species) feel above

214 the global SM-SO axis (where SMA model residuals $\geq 0$ ) while only four fell below the

215 primary SM-SO axis (Fig. 1). In comparison, $46.1 \%$ and $53.9 \%$ of the $n=1190$ wild plant

216 species were approximately evenly distributed above and below the primary SM-SO

217 model, respectively (Figs. 1 and 2).

218 Across all 1231 species, SMA model residuals ranged from -8.4 to 11.7 (average

219 residual=1.14e-11 \pm 2.5 (S.D.)). While accounting for the phylogenetic non-independence

220 of data (Figs. S1 and S2) and uneven sample sizes, the extent of divergence from the SM-

221 SO axis differed significantly across crop types (mixed-effects model $F_{4,711}=13.1$,

$222 p<0.001$, Fig. 2). Specifically, SMA residuals for seed- and tree crops were significantly

223 greater than zero $(p \leq 0.001)$, while residuals for non-seed/ tree crops and wild plant

224 species did not differ from zero ( $p \geq 0.067$, Table $\mathrm{S} 1)$.

225 There was a gradation of divergence from a central SM-SO trade-off (Fig. 2).

226 Tree crops and seed crops expressed the highest SM for a given SO, and in comparison

227 non-seed crops and wild plant expressed lower SM for a given SO; these groups broadly

228 differed significantly from one another in terms of average divergence from a central SM-

229 SO axis (Table S1). Crops harvested for seeds diverged significantly more strongly from

230 a central SM-SO trade-off as compared to wild plants (post-hoc contrast $p \leq 0.0001$; Table

231 S2). Furthermore, 17 seed crops expressed higher average SMA residuals (3.4 \pm 1.6 (S.D.),

232 in comparison to the average SMA residuals observed in 21 non-seed crops $(1.6 \pm 1.8$

233 (S.D.) (Fig. 2, Table S2) The three tree crops in the dataset differed most strongly from a

234 central SM-SO trade-off, showing SMA residuals which were significantly higher than

235 all other crops and wild plants (8.4 \pm 1.8 (S.D.); post-hoc $p \leq 0.04$ for all three contrasts;

236 Fig. 2). Among seed crops, notable divergences from the global SM-SO model included

237 hazelnut (Corylus avellana, SMA residual=7.3), maize (Zea maize, SMA residual=5.1),

238 and sunflower (Helianthus annuus, SMA residual=4.8).

240 Within-crop seed mass seed output trade-offs

241 Data from soy $(n=45)$ and maize $(n=35)$ indicated that SM-SO patterns differed

242 both between these crops, as well as in comparison to a global SM-SO pattern. In maize

$243 \mathrm{SM}$ and SO covaried significantly among individual plants (SMA $r^{2}=0.334, p<0.0001$; 
244 Fig. 3), however artificial selection has resulted in this relationship being positive and

245 therefore opposite that of a global SM-SO pattern (maize SMA slope $=0.27$, 95\% C.I. $=$

$2460.20,0.37$, Fig. 3). Alternatively, soy data indicate that targeted selection for higher SM

247 alone has resulted in a decoupling of SM and SO (Fig. 3). These two seed traits were not

248 significantly related in soy, expressing only a weak negative relationship (SMA slope=-

$2490.14,95 \%$ C.I. $=-0.2,-0.11$, SMA $r^{2}=0.002, p=0.757$; Fig. 3). Consistent with

250 reproductive allocation theory and targeted selection for SM(Sadras 2007), the lack of a

251 significant SM-SO relationship in soy was qualitatively associated with variation in SM

252 which was nearly an order of magnitude lower than variation in SO (where CV=9.5 and

25376.3 , respectively; Table S1).

Phylogenetic signal along the seed mass-seed output trade-off

Across the entire dataset $(n=1231)$ both SM and SO expressed significant phylogenetic signal, with Pagel's $\lambda$ of 0.995 and 0.853 , respectively $(p<0.001$ in both

258 cases, Fig. 4). When crops are removed from the dataset, SM and SO are better predicted

259 by phylogenetic relatedness than when artificially crops are present. Specifically,

260 excluding crops from the datasets resulted in a small, albeit detectable, increase in Pagel's

$261 \lambda$ for both SM (0.996 with crops) and SO (0.859 with crops).

\section{Discussion}

264 The functional profile of crops vs. wild plants

Research documenting the traits that have been targeted by crop domestication

266 suggests that SM and/or SO are among those under the most intensive artificial selection

267 (Meyer et al. 2012). While much of this research has taken archaeological and/or genetic

268 approaches the analysis presented here refines a functional trait-based signature of crop

269 domestication and artificial selection. Specifically, consistent with my hypotheses i)

270 artificial selection has led to most crops significantly deviating from a global SM-SO

271 trade-off (Fig. 1), such that ii) crops under selection for seeds deviate most strongly as

272 compared to non-seed crops (Fig. 2). Yet within seed crops the degree to which artificial

273 selection has reshaped the evolved biomechanical and/ or physiological constraints on

274 trait syndromes (or trait trade-offs), depends on the specific reproductive traits/ yield 
275 components being targeted (Fig. 3). In certain crops such as maize, artificial selection has

276 fundamentally altered the direction of trait trade-offs imposed by natural selection, while

277 in others such as soy artificial selection may only act to decouple the covariation among

278 reproductive traits (Fig. 3)

279 When taken with studies examining other suites of crop functional traits and trait

280 spectra (Milla et al. 2014, Martin et al. 2017), the shifts in SM-SO trade-offs in crops vs.

281 wild plants observed here (Figs. 1-3) represent components of a broader "disruption" in

282 plant resource-use syndromes incurred by artificial selection (Milla et al. 2014). Other

283 and observational data indicate that crops generally express greater values of resource

284 capture traits as compared to their wild progenitors. Indeed, detailed analyses of leaf traits

285 indicate that certain crops express among the most extreme "resource-acquiring" trait

286 syndromes in plants globally (Martin et al. 2018), while at the same time artificial

287 selection has resulted in trait relationships, or "phenotypic integration"(Milla et al. 2014),

288 that are considerably weaker in crops vs. wild plants (Milla et al. 2014, Martin et al.

289 2017).

290 While neither SM nor SO values in crops have been shifted to extreme ends of a

291 reproduction trait spectrum (Fig. 4), the analysis here indicates that relationships between

292 SM and SO within individual species may be fundamentally rewired through

293 domestication (Fig. 3). Our results and other studies exploring trait syndrome disruption

294 (e.g. Milla et al. 2014) provide compelling evidence to indicate crops fundamentally

295 differ from wild plants along a global spectrum of plant form and function(Diaz et al.

296 2016). Quantifying the position of crops and how their traits trade-off along a global trait

297 spectra (Wright et al. 2004, Diaz et al. 2016) represents a means of synthetically defining

298 the functional ecology of crops, which in turn would support a range of hypotheses on the

299 unintended impacts of artificial selection.

301 Conscious and unconscious selection and trait trade-offs in crops

302 Studies employing quantitative trait locus (QTL) mapping would suggest that the

303 results here deviations of crops away from a central SM-SO trade-off (Fig. 1), have likely

304 occurred largely in response to "conscious selection" for these individual traits (e.g. Tao

305 et al. 2017). Major shifts in the shape of the SM-SO relationship in the two crops 
306 explored here is also consistent with strong conscious selection for these particular traits

307 (Fig. 3). Furthermore, analyses of phylogenetic patterns in seed traits here demonstrated a

308 small albeit detectable reduction in phylogenetic signal when crops are removed from

309 analyses (Fig. 4). Major deviations in SM and SO among crops vs. wild relatives, both

310 here and in experimental studies, are most likely consistent with long-term conscious

311 selection of seed traits.

312 At the same time though, theories from functional trait-based ecology do

313 hypothesize that unconscious selection has also played a role in reshaping SM-SO

314 relationships in crops. Specifically, from a plant resource allocation/ natural selection

315 perspective, higher SM at a given SO in open cultivated agricultural environments would

316 be expected to competitive benefits to crop plants, including greater seedling growth rates

317 and survival at greater burial depths. Yet while this theory derived from plant resource

318 allocation theory suggests that unconscious selection may also have contributed to the

319 patterns observed here, experimental evidence in support of this hypothesis is currently

320 lacking (reviewed by Milla et al. 2015). Detailed partitioning of the relative importance

321 of conscious vs. unconscious selection remains a leading avenue for better understanding

322 the genetic vs. phenotypic controls on SM, SO, and their relationships in crops.

\section{References}

Andrade, F. H., V. O. Sadras, C. R. C. Vega, and L. Echarte. 2005. Physiological determinants of crop growth and yield in maize, sunflower and soybean. Journal of Crop Improvement 14:51-101.

Brown, P. J., N. Upadyayula, G. S. Mahone, F. Tian, P. J. Bradbury, S. Myles, J. B. Holland, S. Flint-Garcia, M. D. McMullen, E. S. Buckler, and T. R. Rocheford. 2011. Distinct genetic architectures for male and female inflorescence traits of maize. Plos Genetics 7.

Cayuela, L., A. Stein, and J. Oksanen. 2017. Taxonstand: Taxonomic Standardization of Plant Species Names. R package version 2.1. https://CRAN.Rproject.org/package=Taxonstand.

Cornelissen, J. H. C. 1999. A triangular relationship between leaf size and seed size among woody species: allometry, ontogeny, ecology and taxonomy. Oecologia 118:248-255.

Delignette-Muller, M. L., and C. Dutang. 2015. fitdistrplus: an R package for fitting distributions. Journal of Statistical Software 64:1-34.

Diaz, S., J. Kattge, J. H. C. Cornelissen, I. J. Wright, S. Lavorel, S. Dray, B. Reu, M. Kleyer, C. Wirth, I. C. Prentice, E. Garnier, G. Bonisch, M. Westoby, H. Poorter, P. B. Reich, A. T. Moles, J. Dickie, A. N. Gillison, A. E. Zanne, J. Chave, S. J. 
Wright, S. N. Sheremet'ev, H. Jactel, C. Baraloto, B. Cerabolini, S. Pierce, B. Shipley, D. Kirkup, F. Casanoves, J. S. Joswig, A. Gunther, V. Falczuk, N. Ruger, M. D. Mahecha, and L. D. Gorne. 2016. The global spectrum of plant form and function. Nature 529:167-171.

FAO. 2018. http://www.fao.org/economic/ess/ess-standards/commodity/en/.

Gastauer, M., and J. A. A. Meira-Neto. 2016. An enhanced calibration of a recently released megatree for the analysis of phylogenetic diversity. Brazilian Journal of Biology 76:619-628.

Harper, J. L., P. H. Lovell, and K. G. Moore. 1970. The shapes and sizes of seeds. Annual Review of Ecology and Systematics 1:327-356.

Hayes, F. J., S. W. Buchanan, B. Coleman, A. M. Gordon, P. B. Reich, N. V. Thevathasan, I. J. Wright, and A. R. Martin. 2018. Intraspecific variation in soy across the leaf economics spectrum. Annals of Botany:mcy147.

Hufford, M. B., X. Xu, J. van Heerwaarden, T. Pyhajarvi, J. M. Chia, R. A. Cartwright, R. J. Elshire, J. C. Glaubitz, K. E. Guill, S. M. Kaeppler, J. S. Lai, P. L. Morrell, L. M. Shannon, C. Song, N. M. Springer, R. A. Swanson-Wagner, P. Tiffin, J. Wang, G. Y. Zhang, J. Doebley, M. D. McMullen, D. Ware, E. S. Buckler, S. Yang, and J. Ross-Ibarra. 2012. Comparative population genomics of maize domestication and improvement. Nature Genetics 44:808-U118.

Kattge, J., S. Diaz, S. Lavorel, C. Prentice, P. Leadley, G. Bonisch, E. Garnier, M. Westoby, P. B. Reich, I. J. Wright, J. H. C. Cornelissen, C. Violle, S. P. Harrison, P. M. van Bodegom, M. Reichstein, B. J. Enquist, N. A. Soudzilovskaia, D. D. Ackerly, M. Anand, O. Atkin, M. Bahn, T. R. Baker, D. Baldocchi, R. Bekker, C. C. Blanco, B. Blonder, W. J. Bond, R. Bradstock, D. E. Bunker, F. Casanoves, J. Cavender-Bares, J. Q. Chambers, F. S. Chapin, J. Chave, D. Coomes, W. K. Cornwell, J. M. Craine, B. H. Dobrin, L. Duarte, W. Durka, J. Elser, G. Esser, M. Estiarte, W. F. Fagan, J. Fang, F. Fernandez-Mendez, A. Fidelis, B. Finegan, O. Flores, H. Ford, D. Frank, G. T. Freschet, N. M. Fyllas, R. V. Gallagher, W. A. Green, A. G. Gutierrez, T. Hickler, S. I. Higgins, J. G. Hodgson, A. Jalili, S. Jansen, C. A. Joly, A. J. Kerkhoff, D. Kirkup, K. Kitajima, M. Kleyer, S. Klotz, J. M. H. Knops, K. Kramer, I. Kuhn, H. Kurokawa, D. Laughlin, T. D. Lee, M. Leishman, F. Lens, T. Lenz, S. L. Lewis, J. Lloyd, J. Llusia, F. Louault, S. Ma, M. D. Mahecha, P. Manning, T. Massad, B. E. Medlyn, J. Messier, A. T. Moles, S. C. Muller, K. Nadrowski, S. Naeem, U. Niinemets, S. Nollert, A. Nuske, R. Ogaya, J. Oleksyn, V. G. Onipchenko, Y. Onoda, J. Ordonez, G. Overbeck, W. A. Ozinga, S. Patino, S. Paula, J. G. Pausas, J. Penuelas, O. L. Phillips, V. Pillar, H. Poorter, L. Poorter, P. Poschlod, A. Prinzing, R. Proulx, A. Rammig, S. Reinsch, B. Reu, L. Sack, B. Salgado-Negre, J. Sardans, S. Shiodera, B. Shipley, A. Siefert, E. Sosinski, J. F. Soussana, E. Swaine, N. Swenson, K. Thompson, P. Thornton, M. Waldram, E. Weiher, M. White, S. White, S. J. Wright, B. Yguel, S. Zaehle, A. E. Zanne, and C. Wirth. 2011. TRY - a global database of plant traits. Global Change Biology 17:2905-2935.

Leishman, M. R. I., I. J. Wright, A. T. Moles, and M. Westoby. 2000. The evolutionary ecology of seed size. Pages 31-57 Seeds: the ecology of regeneration in plant communities 2 . 
Lenth, R. V. 2016. Least-squares means: the R package lsmeans. Journal of Statistical Software 69:1-33.

Libenson, S., V. Rodriguez, M. L. Pereira, R. A. Sanchez, and J. J. Casal. 2002. Low red to far-red ratios reaching the stem reduce grain yield in sunflower (vol 42, pg 1180, 2001). Crop Science 42:1761-1761.

Liu, B., T. Fujita, Z. H. Yan, S. Sakamoto, D. Xu, and J. Abe. 2007. QTL mapping of domestication-related traits in soybean (Glycine max). Annals of Botany 100:1027-1038.

Lloyd, D. G. 1987. Selection of offspring size at independence and other size-versusnumber strategies. American Naturalist 129:800-817.

Maddonni, G. A., and M. E. Otegui. 2006. Intra-specific competition in maize: contribution of extreme plant hierarchies to grain yield, grain yield components and kernel composition. Field Crops Research 97:155-166.

Martin, A. R., C. E. Hale, B. E. L. Cerabolini, J. H. C. Cornelissen, J. Craine, W. A. Gough, J. Kattge, and C. K. Tirona. 2018. Inter- and intraspecific variation in leaf economics traits in wheat and maize. Aob Plants 10:ply006.

Martin, A. R., and M. E. Isaac. 2015. Plant functional traits in agroecosystems: a blueprint for research. Journal of Applied Ecology 52:1425-1435.

Martin, A. R., B. Rapidel, O. Roupsard, K. Van den Meersche, E. de M. Virginio Filho, M. Mirna Barrios, and M. E. Isaac. 2017. Intraspecific trait variation across multiple scales: the Leaf Economics Spectrum in coffee. Functional Ecology 31:604-612.

Mayer, L. I., M. A. Rossini, and G. A. Maddonni. 2012. Inter-plant variation of grain yield components and kernel composition of maize crops grown under contrasting nitrogen supply. Field Crops Research 125:98-108.

Meyer, R. S., A. E. DuVal, and H. R. Jensen. 2012. Patterns and processes in crop domestication: an historical review and quantitative analysis of 203 global food crops. New Phytologist 196:29-48.

Milla, R., J. Morente-Lopez, J. M. Alonso-Rodrigo, N. Martin-Robles, and F. S. Chapin. 2014. Shifts and disruptions in resource-use trait syndromes during the evolution of herbaceous crops. Proceedings of the Royal Society B 281.

Milla, R., C. P. Osborne, M. M. Turcotte, and C. Violle. 2015. Plant domestication through an ecological lens. Trends in Ecology \& Evolution 30:463-469.

Moles, A. T., D. D. Ackerly, C. O. Webb, J. C. Tweddle, J. B. Dickie, and M. Westoby. 2005. A brief history of seed size. Science 307:576-580.

Moles, A. T., D. S. Falster, M. R. Leishman, and M. Westoby. 2004. Small-seeded species produce more seeds per square metre of canopy per year, but not per individual per lifetime. Journal of Ecology 92:384-396.

Moles, A. T., and M. Westoby. 2003. Latitude, seed predation and seed mass. Journal of Biogeography 30:105-128.

Moles, A. T., and M. Westoby. 2004. Seedling survival and seed size: a synthesis of the literature. Journal of Ecology 92:372-383.

Pagel, M. 1999. Inferring the historical patterns of biological evolution. Nature 401:877884.

Pinheiro, J., D. Bates, S. DebRoy, D. Sarkar, and R. C. Team. 2016. nlme: Linear and Nonlinear Mixed Effects Models. R package version 3.1-127. 
434

435

436

437

438

439

440

441

442

443

444

445

446

447

448

449

450

451

452

453

454

455

456

457

458

459

460

461

462

463

464

465

466

467

468

469

470

471

472

473

474

475

476

477

478

Revell, L. J. 2012. phytools: an R package for phylogenetic comparative biology (and other things). Methods in Ecology and Evolution 3:217-223.

Sadras, V. O. 2007. Evolutionary aspects of the trade-off between seed size and number in crops. Field Crops Research 100:125-138.

Smith, C. C., and S. D. Fretwell. 1974. The optimal balance between size and number of offspring. The American Naturalist 108:499-506.

Tao, Y. F., E. S. Mace, S. S. Tai, A. Cruickshank, B. C. Campbell, X. R. Zhao, E. J. Van Oosterom, I. D. Godwin, J. R. Botella, and D. R. Jordan. 2017. Whole-genome analysis of candidate genes associated with seed size and weight in Sorghum bicolor reveals signatures of artificial selection and insights into parallel domestication in cereal crops. Frontiers in Plant Science 8.

Thompson, K., S. R. Band, and J. G. Hodgson. 1993. Seed size and shape predict persistence in soil. Functional Ecology 7:236-241.

Wang, E., J. Wang, X. D. Zhu, W. Hao, L. Y. Wang, Q. Li, L. X. Zhang, W. He, B. R. Lu, H. X. Lin, H. Ma, G. Q. Zhang, and Z. H. He. 2008. Control of rice grainfilling and yield by a gene with a potential signature of domestication. Nature Genetics 40:1370-1374.

Warton, D. I., R. A. Duursma, D. S. Falster, and S. Taskinen. 2012. smatr 3-an R package for estimation and inference about allometric lines. Methods in Ecology and Evolution 3:257-259.

Webb, C. O., D. D. Ackerly, and S. W. Kembel. 2008. Phylocom: software for the analysis of phylogenetic community structure and trait evolution. Bioinformatics 24:2098-2100.

Webb, C. O., and M. J. Donoghue. 2005. Phylomatic: tree assembly for applied phylogenetics. Molecular Ecology Notes 5:181-183.

Westoby, M. 1998. A leaf-height-seed (LHS) plant ecology strategy scheme. Plant and Soil 199:213-227.

Westoby, M., D. S. Falster, A. T. Moles, P. A. Vesk, and I. J. Wright. 2002. Plant ecological strategies: Some leading dimensions of variation between species. Annual Review of Ecology and Systematics 33:125-159.

Westoby, M., E. Jurado, and M. Leishman. 1992. Comparative evolutionary ecology of seed size. Trends in Ecology \& Evolution 7:368-372.

Westoby, M., M. Leishman, and J. Lord. 1996. Comparative ecology of seed size and dispersal. Philosophical Transactions of the Royal Society B-Biological Sciences 351:1309-1317.

Wickham, H. 2017. modelr: Modelling Functions that Work with the Pipe. R package version 0.1.1. https://CRAN.R-project.org/package=modelr.

Wikstrom, N., V. Savolainen, and M. W. Chase. 2001. Evolution of the angiosperms: calibrating the family tree. Proceedings of the Royal Society B-Biological Sciences 268:2211-2220.

Wright, I. J., P. B. Reich, M. Westoby, D. D. Ackerly, Z. Baruch, F. Bongers, J. Cavender-Bares, T. Chapin, J. H. C. Cornelissen, M. Diemer, J. Flexas, E. Garnier, P. K. Groom, J. Gulias, K. Hikosaka, B. B. Lamont, T. Lee, W. Lee, C. Lusk, J. J. Midgley, M. L. Navas, U. Niinemets, J. Oleksyn, N. Osada, H. Poorter, P. Poot, L. Prior, V. I. Pyankov, C. Roumet, S. C. Thomas, M. G. Tjoelker, E. J. 

428:821-827.

481 Zhou, Z. K., Y. Jiang, Z. Wang, Z. H. Gou, J. Lyu, W. Y. Li, Y. J. Yu, L. P. Shu, Y. J. Zhao, Y. M. Ma, C. Fang, Y. T. Shen, T. F. Liu, C. C. Li, Q. Li, M. Wu, M. Wang, Y. S. Wu, Y. Dong, W. T. Wan, X. Wang, Z. L. Ding, Y. D. Gao, H. Xiang, B. G. Zhu, S. H. Lee, W. Wang, and Z. X. Tian. 2015. Resequencing 302 wild and cultivated accessions identifies genes related to domestication and improvement in soybean. Nature Biotechnology 33:408-U125. 


\section{$488 \quad$ Figures}

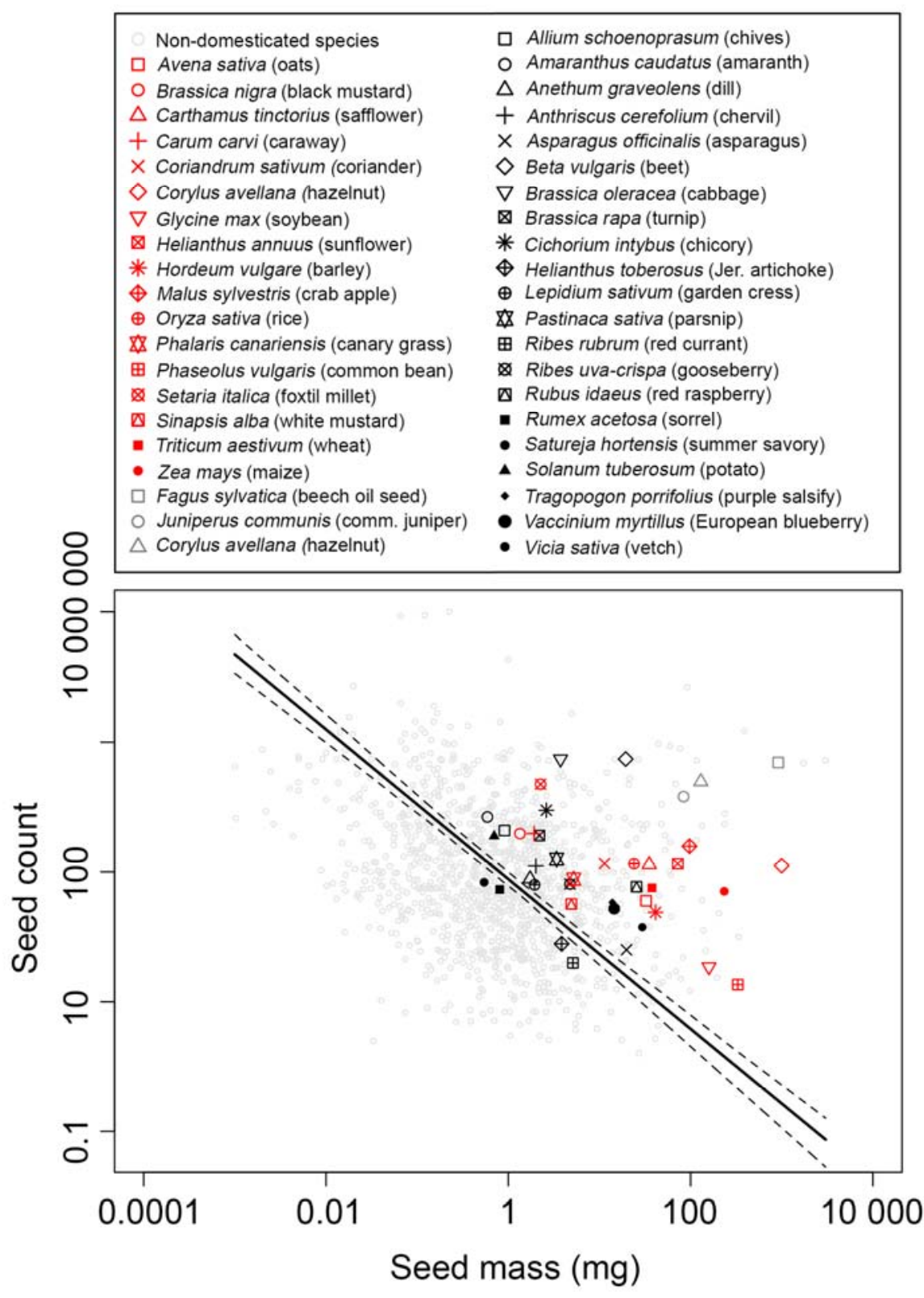

Avena sativa (oats)

+ Carum carvi (caraway)

Coriandrum sativum (coriander)

Helianthus annuus (sunflower)

ordeum vulgare (barley)

P Phaseolus vulgaris (common bean)

Setaria italica (foxtil millet)

- Juniperus communis (comm. juniper)

Corylus avellana (hazelnut)
Allium schoenoprasum (chives)

- Amaranthus caudatus (amaranth)

Satureja hortensis (summer savory)

- Solanum tuberosum (potato)

- Tragopogon porrifolius (purple salsify)

- Vaccinium myrtillus (European blueberry)

cia sativa (vetch)

491 Fig. 1. Relationship between seed mass and seed output across $n=1190$ non-domesticated

492 species and $n=41$ of the world's most widespread crops. Solid black trend line represents

493 a standardized major axis regression fit across the entire dataset $(n=1231)$ and dashed

494 lines represent $95 \%$ confidence limits. 


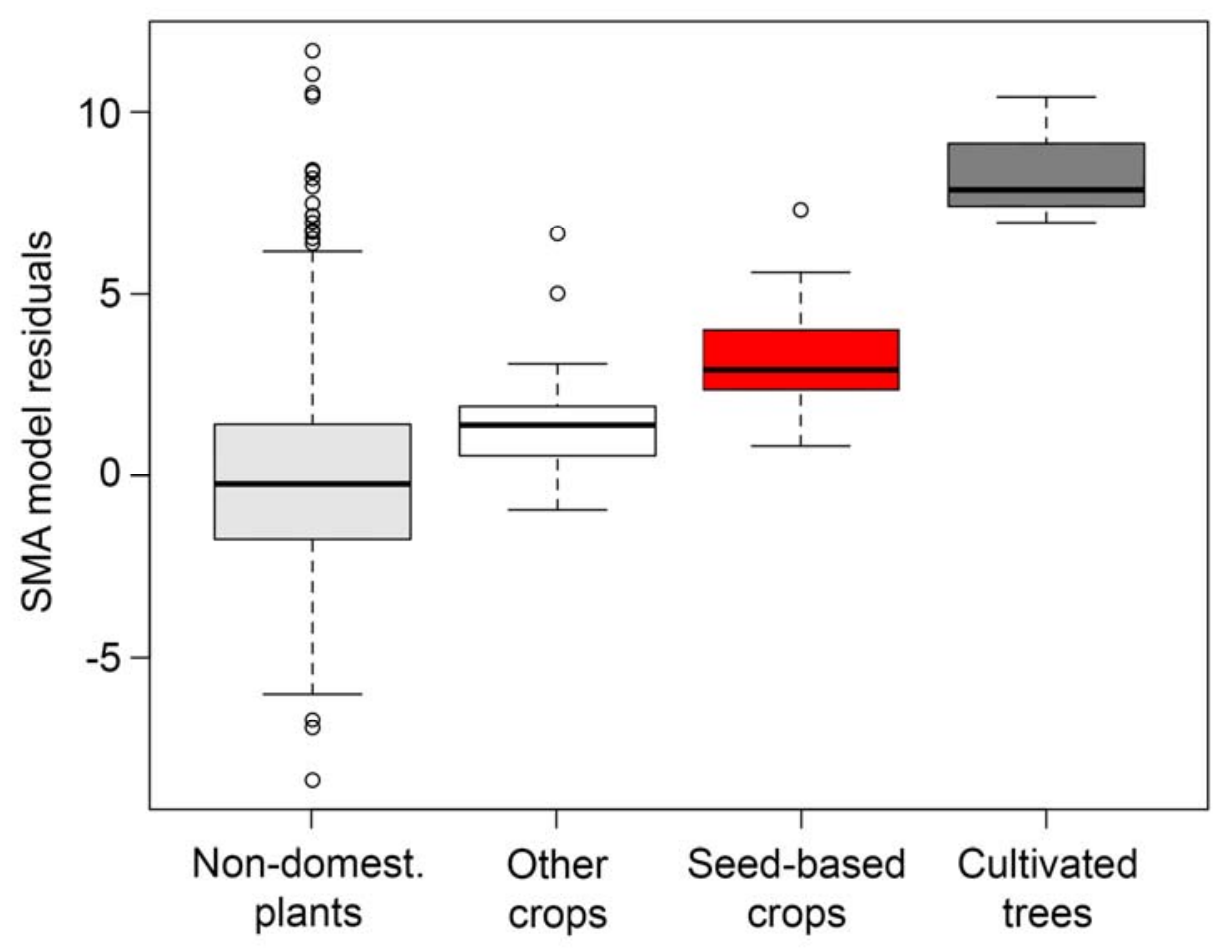

497 Fig. 2. Deviations from a global seed mass-seed output trade-off in three types of crops

498 and non-domesticated plants. Deviations from a global SM-SO relationship are calculated 499 as residuals from a standardized major axis regression fit to $n=1231$ plant species (see 500 Fig. 1). "Other crops" $(n=21)$ correspond to plant species cultivated for vegetative- or 501 belowground structures. Sample sizes for seed-based crops, cultivated trees, and non-

502 domesticated plants are $n=17, n=3$, and $n=1190$, respectively. 


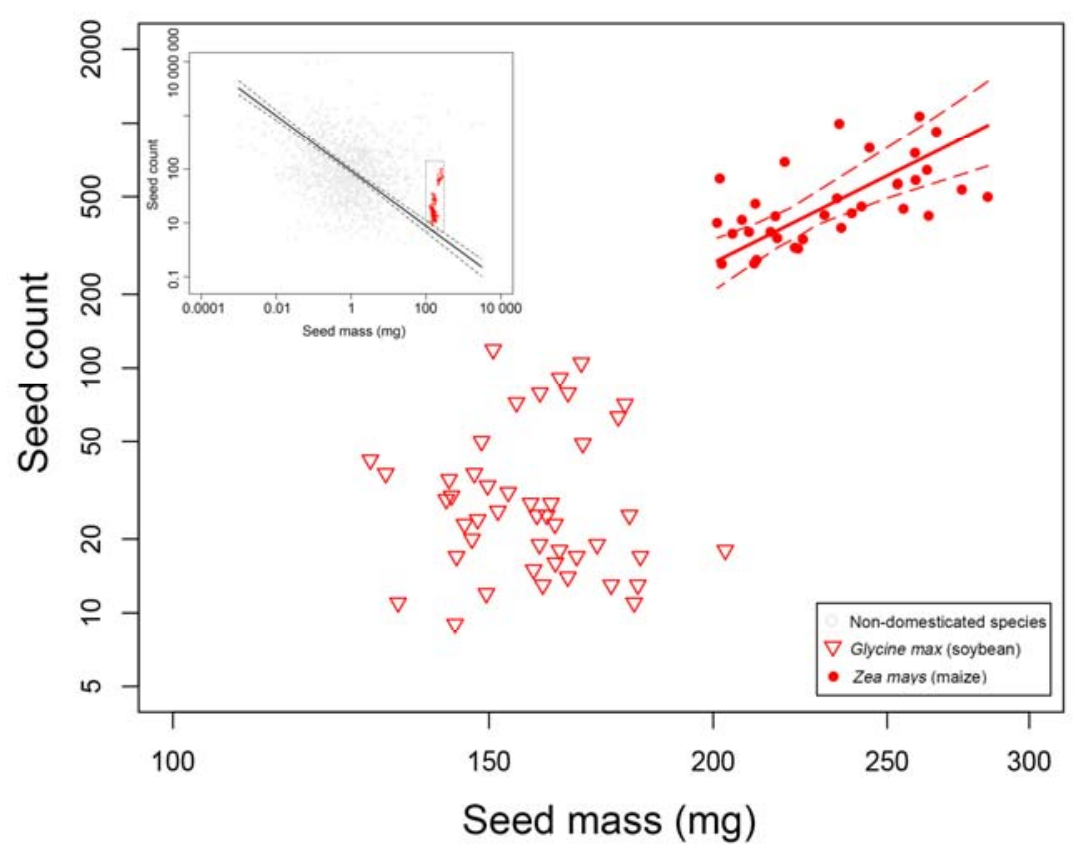

504

505 Fig. 3. Seed mass and seed output in soybean (Glycine max, $n=45)$ and maize (Zea maize, $506 n=37)$. Data are presented in relation to a SM-SO trade-off observed across 1231 plant

507 species (gray rectangle, inset graph). Solid red trend line represents a standardized major 508 axis (SMA) regression fit to the maize data with red dashed lines representing $95 \%$

509 confidence limits. The SMA model fit to soy data was not significant, so is not presented 510 here. 

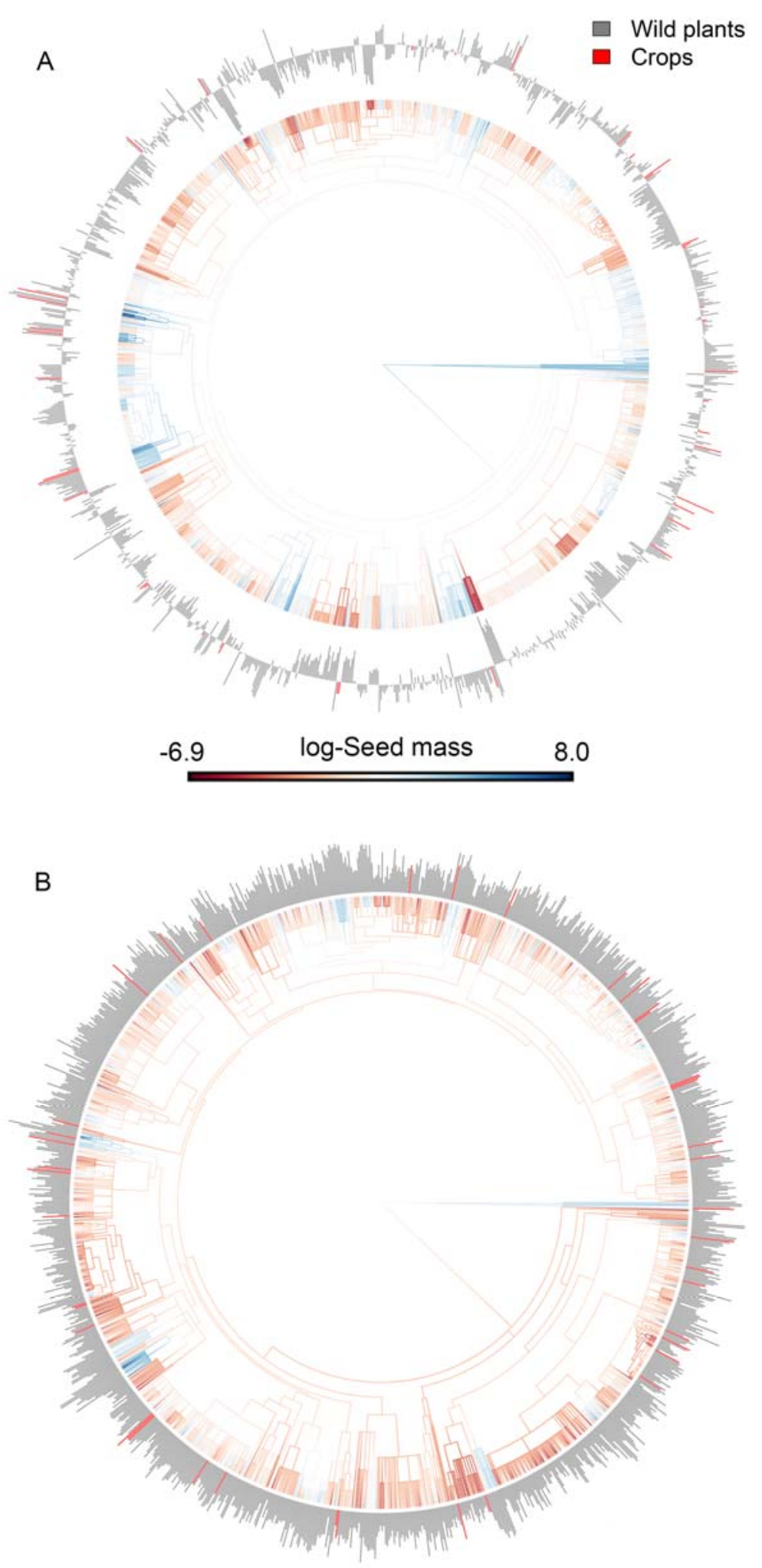

0.5 log-Seed count 16.1

\section{1}


512 Fig. 4. Variation in seed mass (A) and seed output (B) across a phylogeny of 1,231 crop-

513 and wild plant species. Bars across the tips of the phylogeny represent mean seed mass

514 and seed count ( plant $^{-1}$ ) values, with crop species highlighted in red and wild plants in

515 gray. 